\title{
Neuroprotective Role of Trans-Resveratrol in a Murine Model of Familial Alzheimer's Disease
}

\author{
David Porquet $^{\mathrm{a}, \mathrm{e}}$, Christian Griñán-Ferréa Isidre Ferrer $^{\mathrm{d}, \mathrm{e}}$, Antoni Camins $^{\mathrm{a}, \mathrm{e}}$, Coral Sanfeliu ${ }^{\mathrm{b}}$, \\ Jaume del Valle ${ }^{\mathrm{a}, \mathrm{c}, \mathrm{e}}$ and Mercè Pallàs ${ }^{\mathrm{a}, \mathrm{e}, *}$ \\ ${ }^{a}$ Secció de Farmacologia, Departament Farmacologia i Química Terapéutica, Facultat de Farmácia, Institut de \\ Biomedicina, Universitat de Barcelona, Barcelona, Spain \\ ${ }^{\mathrm{b}}$ Institut d'Investigacions Biomèdiques de Barcelona, CSIC, IDIBAPS, Barcelona, Spain \\ ${ }^{\mathrm{c}}$ Grup de Neuroplasticitat i Regeneració, Institut de Neurociències i Departament de Biologia Cellular, Fisiologia \\ i Immunologia, Universitat Autònoma de Barcelona, Bellaterra, Spain \\ dinstitut de Neuropatologia de l'Hospital Universitari de Bellvitge, Institut d'Investigació Biomédica de Bellvitge, \\ Universitat de Barcelona, Bellvitge, Spain \\ ${ }^{\mathrm{e}}$ Centros de Investigación Biomédica en Red de Enfermedades Neurodegenerativas (CIBERNED), Spain
}

\begin{abstract}
The amyloid- $\beta$ protein precursor/presenilin 1 (AßPP/PS1) mouse model of Alzheimer's disease (AD) has provided robust neuropathological hallmarks of familial AD-like pattern. $\mathrm{AD}$ is a neurodegenerative process that causes severe cognitive impairment; it is characterized by the accumulation of amyloid- $\beta$ (A $\beta$ ) and hyperphosphorylated tau forms and by oxidative and inflammatory processes in brain. Currently, efforts are made to understand biochemical pathways because there is no effective therapy for AD. Resveratrol is a polyphenol that induces expression and activation of several neuroprotective pathways involving Sirtuin1 and AMPK. The objective of this work was to assess the effect of oral resveratrol administration on AßPP/PS1 mice. Long-term resveratrol treatment significantly prevented memory loss as measured by the object recognition test. Moreover, resveratrol reduced the amyloid burden and increased mitochondrial complex IV protein levels in mouse brain. These protective effects of resveratrol were mainly mediated by increased activation of Sirtuin 1 and AMPK pathways in mice. However, an increase has been observed in $I L 1 \beta$ and $T N F$ gene expression, indicating that resveratrol promoted changes in inflammatory processes, although no changes were detected in other key actors of the oxidative stress pathway. Taken together, our findings suggest that resveratrol is able to reduce the harmful process that occurs in A $\beta \mathrm{PP} / \mathrm{PS} 1$ mouse hippocampus, preventing memory loss.
\end{abstract}

Keywords: AMPK, inflammation, mitochondria, resveratrol, sirtuin 1

\section{INTRODUCTION}

A central issue in cognitive neuroscience of research on aging is pinpointing precise neural mechanisms that determine cognitive outcome in late adulthood, as well as identifying early markers of neurodegen-

\footnotetext{
*Correspondence to: Mercè Pallàs, Secció de Farmacologia, Facultat de Farmácia, Universitat de Barcelona, Av. Diagonal 643, Barcelona 08028, Spain. Tel.: +34 934024531; Fax: +34 934035982; E-mail: pallas@ub.edu.
}

eration and preventive strategies to impede cognitive impairment in aging. Alzheimer's disease (AD) is the most prevalent neurodegenerative disorder associated with age and characterized by senile plaques and neurofibrillary tangles. Senile plaques are formed by the accumulation of amyloid- $\beta(A \beta)_{1-40}$ and $A \beta_{1-42}$ peptides, which result from the sequential cleavage of amyloid- $\beta$ protein precursor (A $\beta P P)$ by $\beta$-secretase (BACE) and $\gamma$-secretase. The production of $A \beta$ peptides is prevented by alternative cleavage of A $\beta P P$ by $\alpha$-secretase followed by $\gamma$-secretase. Donmez et al. 
[1] have reported that production of $A \beta$ and plaques in the $3 \times \mathrm{Tg}-\mathrm{AD}$ transgenic mouse model of $\mathrm{AD}$ is reduced by the overexpression of Sirtuin 1 (SIRT1) and is increased by knocking out SIRT1 in brain. SIRT1 directly activates transcription of the gene encoding $\alpha$-secretase, the ADAM10 gene. Additionally, SIRT1 deacetylates and coactivates the Retinoic acid receptor $\beta$, a known regulator of $A D A M 10$ transcription.

It is noteworthy that in $\mathrm{AD}$, mitochondrial function is affected. Reduction of mitochondrial complex IV (MCIV) activity [2, 3] and increased reactive oxygen species production $[4,5]$ was reported elsewhere. Accumulation of mitochondrial DNA changes might increase reactive oxygen species production and reduce mitochondrial adenine triphosphate (ATP) in an age-dependent manner. Increases of somatic mitochondrial DNA in aging might contribute to $\mathrm{AD}$ development [6].

An early-onset form of $\mathrm{AD}$, familial $\mathrm{AD}$ (defined for individuals $<65$ years of age), accounts for $5 \%$ of all cases and is directly linked with highly penetrant autosomal dominant mutations in one of three different genes: the presenilin 1 (PS1) gene; the PS2 gene, or the A $\beta P P$ gene.

Resveratrol, a natural polyphenolic compound found in grapes and red wine, increases metabolic rate, insulin sensitivity, mitochondrial biogenesis, and physical endurance, and also reduces fat accumulation in mice and, at the brain level, is postulated as an option to prevent $\mathrm{AD}$ [7-9]. Although it is thought that resveratrol targets SIRT1, this remains controversial because resveratrol also activates 5-Adenosine monophosphate (AMP)-activated protein kinase (AMPK), which also regulates insulin sensitivity and mitochondrial biogenesis. Um et al. [10] demonstrated that the metabolic effects of resveratrol are dependent on AMPK using knockout mice. In addition, resveratrol, during food deprivation (caloric restriction), causes an increase in the cellular AMP/ATP ratio, resulting in the activation of AMPK, which initiates a signaling process that recruits mediators of oxidative metabolism and mitochondrial biogenesis including PGC1- $\alpha$, the peroxisome proliferator-activated receptor-gamma (PPAR- $\delta$ ), and others [11].

Double-transgenic A $\beta P P / P S 1$ mice express a chimeric mouse/human A $\beta P P$ bearing the Swedish mutation (Mo/Hu AßPP695swe) and a mutant human PS1-dE9, both causative of familial AD. Transgenic mice show senile plaques in cortical and hippocampal areas starting at 4 months [12] and impaired memory and learning performance between 6 and 15 months [13-15]. However, these mice have no alter- ations in motor function or anxiety-related behavior [16]. Moreover, these animals have increased BACE activity [17], decreased ADAM10 expression [18], and decreased synaptosomal synaptophysin [19]. In reference to inflammatory markers $\mathrm{A} \beta \mathrm{PP} / \mathrm{PS} 1$ mice present elevaled levels of IL-1 $\beta$ [20] and TNF [21].

The aim of this work was to elucidate the interplays between the amyloidogenic pathway, SIRT1 and AMPK signaling, and resveratrol as a preventative agent in the mouse familial AD model A $\beta P P / P S 1$. The generation of this knowledge would afford us new clues to confront neurodegenerative disorders with abnormal $\mathrm{A} \beta \mathrm{PP}$ processing, such as familial $\mathrm{AD}$, and to help in preventing the processes linked with this illness.

\section{METHODS}

\section{Animals and resveratrol feeding}

All animal protocols were conducted and approved by the Ethic Committee for Animal Use from the University of Barcelona in accordance with the Generalitat de Catalunya guidelines for the Care and Use of Laboratory Animals. All efforts were made to minimize animal numbers and distress.

AßPPswe/PS1dE9 male mice (2 months of age) that were treated and age-matched control littermates were both fed with mouse chow (Harlam Diet) containing $1 \%$ resveratrol [22] or the same chow without resveratrol ( $n=10$ in each group) for 10 months. Daily resveratrol consumption was calculated to be $4 \mathrm{mg} / \mathrm{kg} /$ day (mean of food intake of $4 \mathrm{~g} / \mathrm{animal} / \mathrm{day}$ ).

\section{Novel object recognition test (NORT)}

Changes in cognition were tested with an objectrecognition task as described elsewhere [23]. Animals were placed in a $90^{\circ}$, two-arm, 25-cm-long, $20-\mathrm{cm}-$ high, and 5-cm-wide black maze. Light intensity in the middle of the field was 30 lux. The objects to be discriminated were made of plastic (object A, $5.25 \mathrm{~cm}$ in height, and object B, $4.75 \mathrm{~cm}$ in height). For the first 3 days, the mice were individually acclimatized to the apparatus for $10 \mathrm{~min}$. On day 4, the animals were submitted to a 10-min acquisition trial (first trial), during which they were placed in the maze in the presence of two identical novel objects $(\mathrm{A}+\mathrm{A}$ or $\mathrm{B}+\mathrm{B})$, which were localized at the end of each arm. A 10-min retention trial (second trial) occurred $2 \mathrm{~h}$ later. During this second trial, objects A and B were placed in the maze, and the time that the animal explored the new object $\left(t_{n}\right)$ and the old object $\left(t_{o}\right)$ were recorded. A Discrim- 
ination index (DI) was defined as $\left(t_{n}-t_{o}\right) /\left(t_{n}+t_{o}\right)$. In order to avoid object preference biases, objects $\mathrm{A}$ and $\mathrm{B}$ were counterbalanced so that one half of the animals in each experimental group were first exposed to object $\mathrm{A}$ and then to object $\mathrm{B}$, whereas the remaining one half first saw object $\mathrm{B}$ and then object $\mathrm{A}$. The maze and the objects were cleaned with $96^{\circ}$ ethanol between experiments to eliminate olfactory cues.

\section{Brain processing}

Animals were anesthetized with $80 \mathrm{mg} / \mathrm{kg}$ of sodium pentobarbital and intracardially perfused with saline serum. Afterward, brains were dissected and separated sagitally into two hemispheres: one for histological staining, and the other, for protein and RNA extraction. Hemispheres for histological staining were frozen by immersion in isopentane, chilled on dry ice, and stored at $-80^{\circ} \mathrm{C}$ until sectioning. Thereafter, the frozen brains were embedded in Optimal cutting temperature (OCT) cryostat-embedding compound (Tissue-Tek; Torrance, CA, USA), cut into $20-\mu \mathrm{m}$-thick sections on a cryostat (Leyca Microsystems, Germany) at $-18^{\circ} \mathrm{C}$, and placed on slides. Slides containing brain sections were fixed with acetone for $10 \mathrm{~min}$ at $4^{\circ} \mathrm{C}$, allowed to dry at room temperature, and then frozen at $-20^{\circ} \mathrm{C}$ until further staining. The remaining hemispheres were dissected and stored at $-80^{\circ} \mathrm{C}$ until protein or RNA extraction.

\section{Thioflavin S staining}

Slides were allowed to defrost at room temperature and then were rehydrated with Phosphate-buffered saline (PBS) for $5 \mathrm{~min}$. Later, the brain sections were incubated with $0.3 \%$ Thioflavin S (Sigma-Aldrich) for $20 \mathrm{~min}$ at room temperature in the dark. Subsequently, these were submitted to washes in 3-min series, specifically with $80 \%$ ethanol (2 washes), $90 \%$ ethanol (1 wash), and 3 washes with PBS. Finally, the slides were mounted using Fluoromount (EMS), allowed to dry overnight at room temperature in the dark, and stored at $4{ }^{\circ} \mathrm{C}$. Image acquisition was performed with an epifluorescence microscope (BX41; Olympus, Germany). For plaque quantification, similar and comparable histological areas were selected, focusing on having the hippocampus and the whole cortical area positioned adjacently.

\section{Protein extraction}

Brains were micronized by freezing with liquid nitrogen and grinding with a mortar. For total pro- tein extraction, lysis buffer $(50 \mathrm{mM}$ Tris- $\mathrm{HCl}, 150 \mathrm{mM}$ $\mathrm{NaCl}, 5$ mM EDTA, $1 \%$ Triton X-100, pH 7.4), EDTAfree Protease inhibitor cocktail (Roche, Mannheim, Germany), and Phosphatase inhibitor cocktail 1 (Sigma-Aldrich, St. Louis, MO, USA) were added to micronized tissue and left on ice for $30 \mathrm{~min}$. Then, the samples were centrifuged at $10,000 \times g$ for $10 \mathrm{~min}$ and a supernatant with total protein content was collected. All of the protein extraction steps were carried out at $4^{\circ} \mathrm{C}$. Protein concentration was determined by the Bradford protein assay.

\section{Western blot}

For western blot analysis, $20 \mu \mathrm{g}$ of protein were denatured at $95^{\circ} \mathrm{C}$ for $5 \mathrm{~min}$ in sample buffer $(0.5 \mathrm{M}$ Tris-HCl, pH 6.8, 10\% glycerol, 2\% Sodium dodecyl sulfate [SDS], 5\% $\beta$-mercaptoethanol, $0.05 \%$ bromophenol blue), separated by Sodium dodecyl sulfatePolyacrylamide gel electrophoresis (SDS-PAGE) onto $8-12 \%$ polyacrylamide gels and transferred onto Immobilon polyvinylidene difluoride membranes (Millipore, Billerica, MA, USA). The membranes were incubated overnight at $4{ }^{\circ} \mathrm{C}$ with the following primary antibodies: anti- $\beta$-actin (Sigma; $1: 10,000)$; anti-Sirt1 (Abcam, 1:1,000); anti-p53 (Abcam, 1:500); anti-acetyl p53 (L382) (Millipore, 1:500); anti-AMPK (Cell Signaling, 1:1,000); anti-pAMPK (Cell Signaling, 1:1,000); OXPHOS cocktail and porin (1:500; MitoSciences); catalase (1:2,000; Calbiochem), and superoxide dismutase (SOD) (1:3000; Calbiochem) diluted with Tris-buffered saline containing $0.1 \%$ Tween 20 (TBS-T) and 5\% Bovine serum albumin (BSA). Membranes were then washed and incubated with secondary antibodies and diluted with TBS-T for $1 \mathrm{~h}$ at room temperature. Protein bands were visualized using a chemiluminescent horseradish peroxidase substrate (Millipore) and ChemiDoc XRS + (Bio$\mathrm{rad}$ ). Band intensities were quantified by densitometric analysis using Image Lab software and values were normalized to $\beta$-actin.

\section{RNA extraction and gene expression determination}

Total RNA isolation was carried out by means of Trizol reagent following the manufacturer's instructions. RNA content in the samples was measured at $260 \mathrm{~nm}$, and the purity of the samples was determined by the A260/280 ratio in a NanoDrop ND-1000 (Thermo Scientific). Samples were also tested in a Bioanalyzer 2100B (Agilent Technologies) to determine the RNA integrity number. 
Reverse transcription-polymerase chain reaction (RT-PCR) was performed as follows: $2 \mu \mathrm{g}$ of messenger RNA (mRNA) was reverse-transcribed using the High Capacity cDNA Reverse Transcription kit (Applied Biosystems). Then, qPCR was performed using TaqMan gene expression assays (Applied Biosystems), doing triplicates for each gene and cDNA sample in 96-well optical plates. The TaqMan probes used were Interleukin-1 $\beta$ (Illb) (Mm00434228_m1), Interleukin-6 (Il6) (Mm00446190_m1), Tumor necrosis factor (Tnf) (Mm00443260_g1), Nuclear factor erythroid 2-related factor 2 (Nfe2l2) (Mm00477784_m1), Heme oxygenase-1 (Hmox) (Mm00516005_m1), and Jun (Jun) (Mm00495062), with TATA-binding protein $(T b p)$ (Mm00446971_m1) as housekeeping. For each $20 \mu$ l of TaqMan reaction, $9 \mu \mathrm{l}$ cDNA $(25 \mathrm{ng})$ was mixed with $1 \mu \mathrm{L} 20 \mathrm{x}$ probe of TaqMan Gene Expression Assays and $10 \mu \mathrm{l}$ of 2x TaqMan Universal PCR Master Mix. The reactions were carried out using the following parameters: $50^{\circ} \mathrm{C}$ for $2 \mathrm{~min} ; 95^{\circ} \mathrm{C}$ for $10 \mathrm{~min}, 40$ cycles at $95^{\circ} \mathrm{C}$ for $15 \mathrm{~s}$, and at $60^{\circ} \mathrm{C}$ for 1 min using the StepOnePlus Real-Time PCR System (Applied Biosystems). Finally, all TaqMan PCR data were normalized to TBP using the delta-delta $\mathrm{Ct}$ method.

\section{Statistical analysis}

Results were analyzed statistically by GraphPad PRISM (GraphPad Software, Inc.) software. Data are presented as mean \pm Standard error of the mean (SEM), and means were compared with two-tailed, unpaired Student $t$-test. In the Object recognition test (ORT), a one-sample $t$-test was used to examine whether single columns were different from zero. Statistical significance was reached when $p$ values were $<0.05$. All the experiments and its statistics were conducted with 7 to 10 animals for each experimental group.

\section{RESULTS}

Resveratrol ameliorated short-term memory in A $\beta P P / P S 1$ mice

To date, it has been found that transgenic mice that overexpress A $\beta P P / P S 1$ show loss of memory [13-15]. ORT demonstrated that at 12 months, A $\beta P P / P S 1$ mice fed with resveratrol chow exhibited significantly improved memory capabilities (Fig. 1A) in comparison to $\mathrm{A} \beta \mathrm{PP} / \mathrm{PS} 1$ control mice.
A

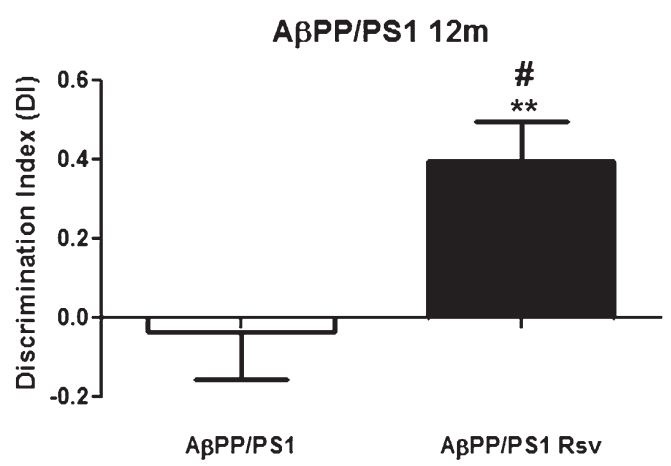

B

Synaptophysin

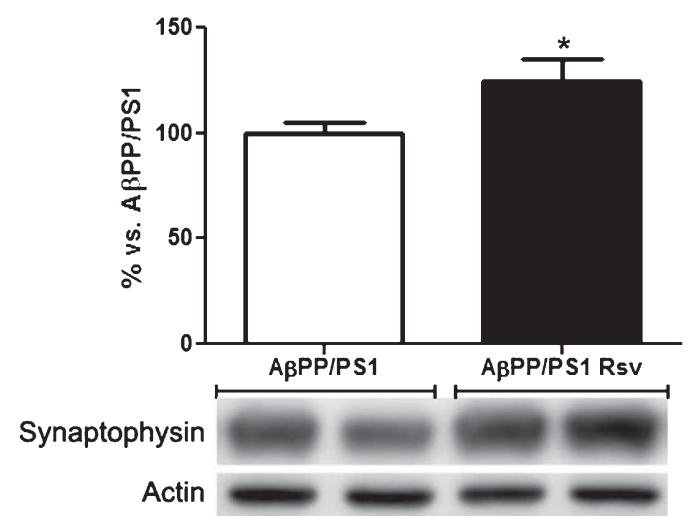

Fig. 1. A) Object recognition test analysis for $A \beta P P / P S$ control and resveratrol-fed mice. Bars represent discrimination index, mean \pm Standard error of the mean (SEM). One sample $t$-test ${ }^{* *} p<0.01$ from zero. Student $t$-test versus control; ${ }^{\#} p<0.05$. B) Western blot analysis for synaptophysin in A $\beta P P / P S 1$ control and resveratrol-fed mice. Results are represented as mean \pm SEM. Student $t$-test versus control; ${ }^{*} p<0.05$.

The previously mentioned beneficial effects of resveratrol were supported by an increase in synaptophysin, a presynaptic protein that reflects an improvement in synaptic activity in resveratrol-fed mice (Fig. 1B).

Resveratrol treatment reduced plaque pathology but did not alter $A \beta P P$ and its carboxy terminal fragments (CTF) in A $\beta P P / P S 1$ mice

To test the effect of resveratrol on amyloid plaque pathology, thioflavin S staining was used. Resveratrol markedly reduced thioflavin S-positive compact plaques compared with control mice (Fig. 2). Quantification of plaques revealed a significant reduction in plaque counts and plaque burden in hippocampus and 
A

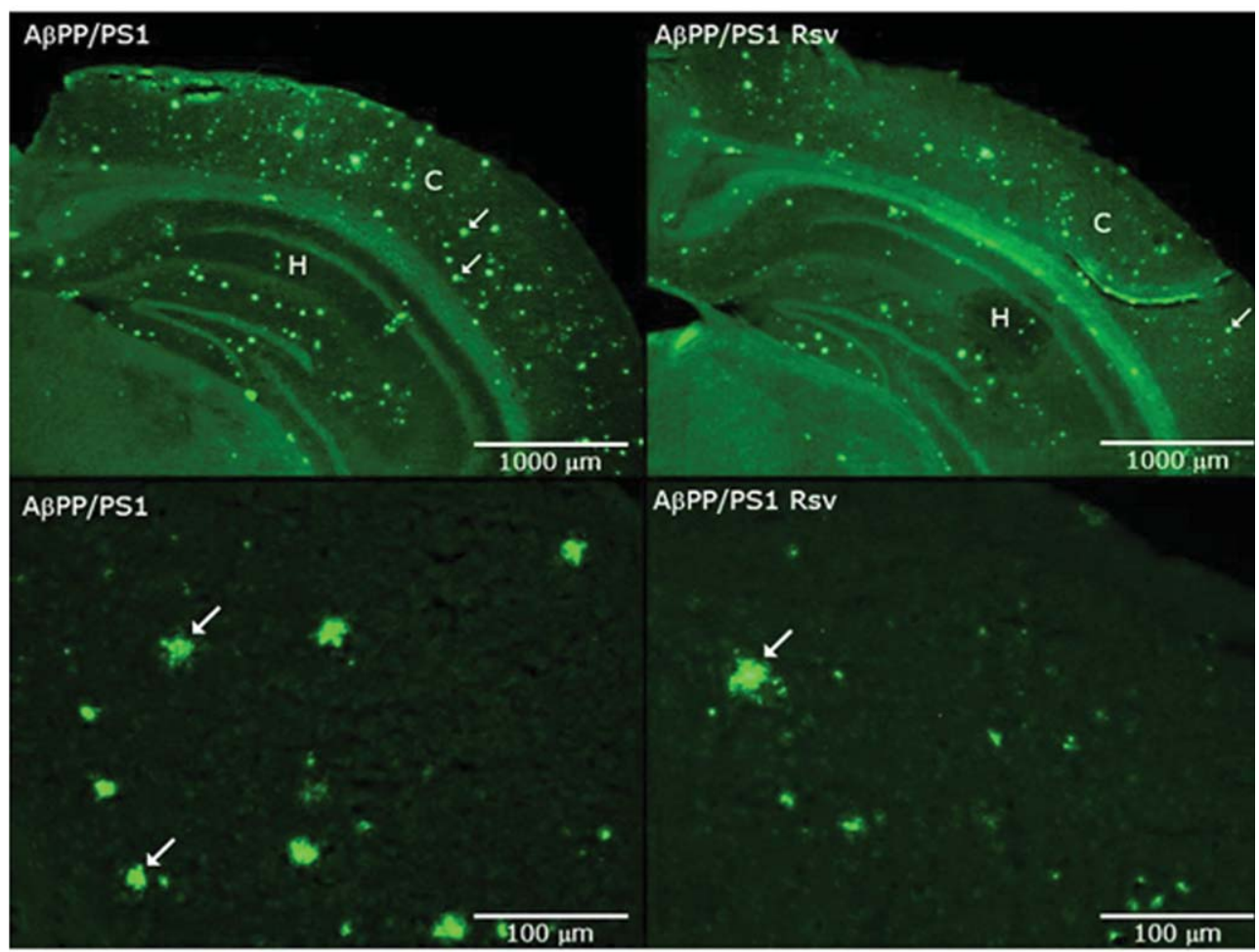

B
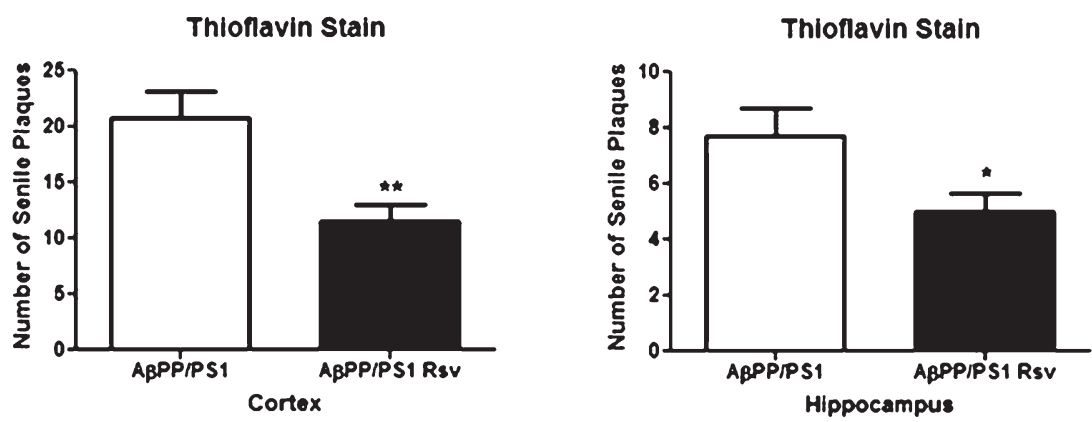

Fig. 2. A) Thioflavin S staining of $A \beta$ plaques in mouse brains. Representative images of histopathological brain state of $A \beta P P / P S 1$ control and resveratrol-fed mice. White arrows are representative indicators of the presence of $A \beta$ plaques in the studied areas, cortex (C), and hippocampus $(\mathrm{H})$. Bar chart showing quantification of the number of amyloid plaques in the cerebral cortex (B) and hippocampus (C) of A $3 P P / P S 1$ control and resveratrol-treated animals. For quantification parameters, see Materials and Methods. Bars represent mean \pm Standard error of the mean (SEM). Student $t$-test $* p<0.05 ; * * p<0.01$ versus control.

medial cortex of resveratrol-fed mice compared with controls (Fig. 2A-C).

To investigate the mechanism responsible for the resveratrol-induced reduction in plaque counts, enzymes that mediate A $\beta P P$ cleavage were determined. We found no changes in PS1, but a significant reduction in BACE and ADAM 10 protein levels were observed (Fig. 3A-C).

Moreover, full-length $\mathrm{A} \beta \mathrm{PP}$ and $\mathrm{A} \beta \mathrm{PP}$ cleavage products $\beta$ CTF (C99) and $\alpha$ CTF (C83) levels were determined by western blot using AßPP G369 antibody (against the A $\beta P P$ cytoplasmic tail). Resveratrol 

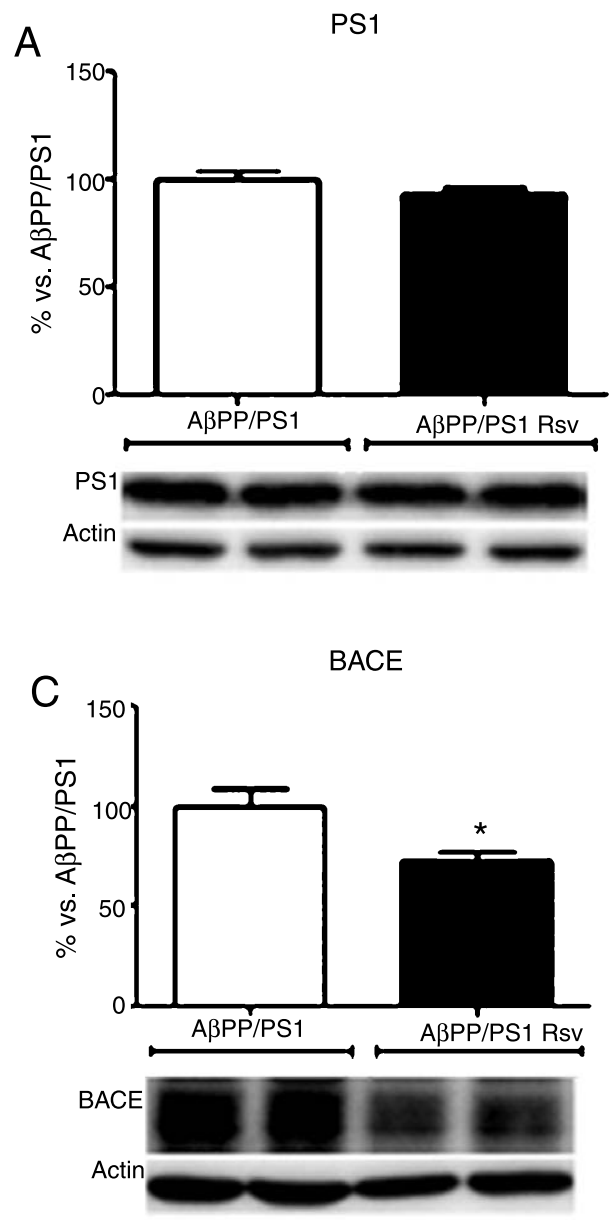

$\mathrm{E}$
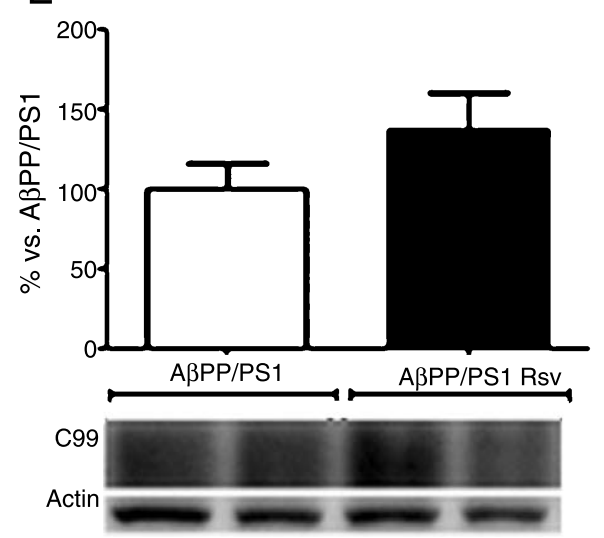

ADAM10
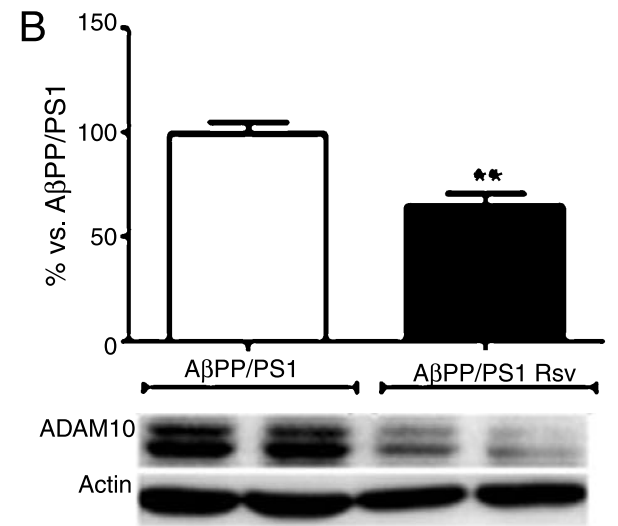

Holo A $\beta P P$

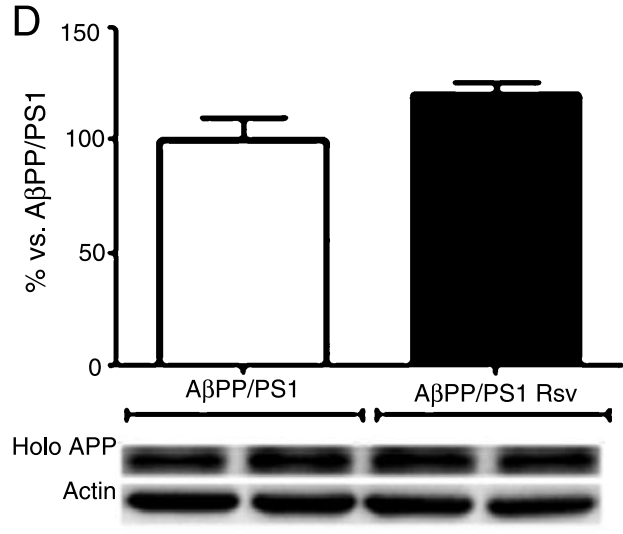

C83

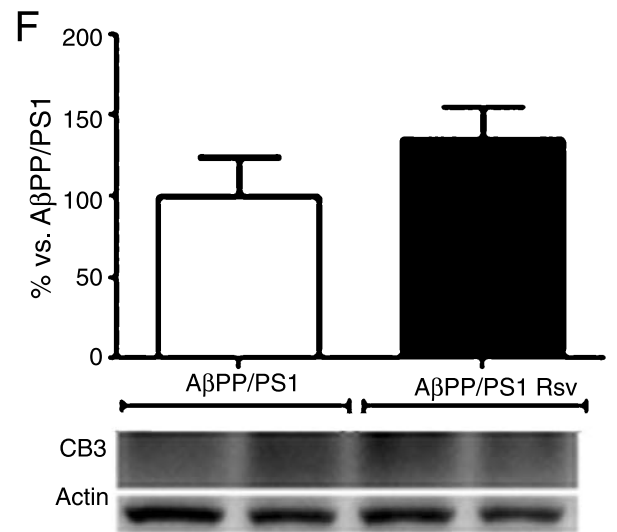

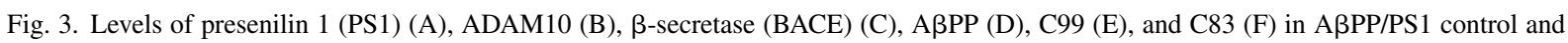
resveratrol-fed mice. Bars represent mean \pm Standard error of the mean (SEM). Student $t$-test: * $p<0.05 ; * * p<0.01$ versus control. 
A

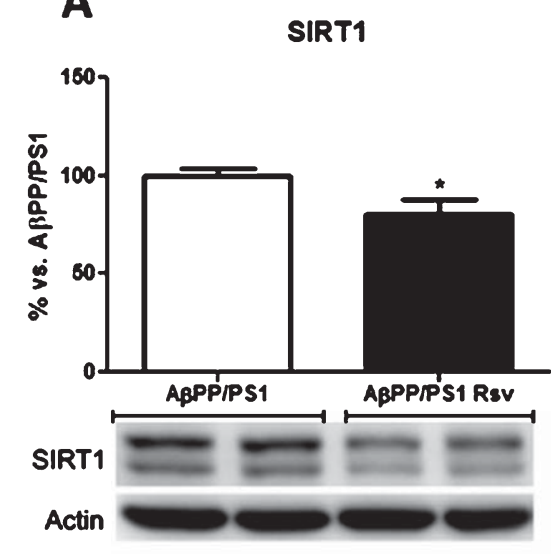

C
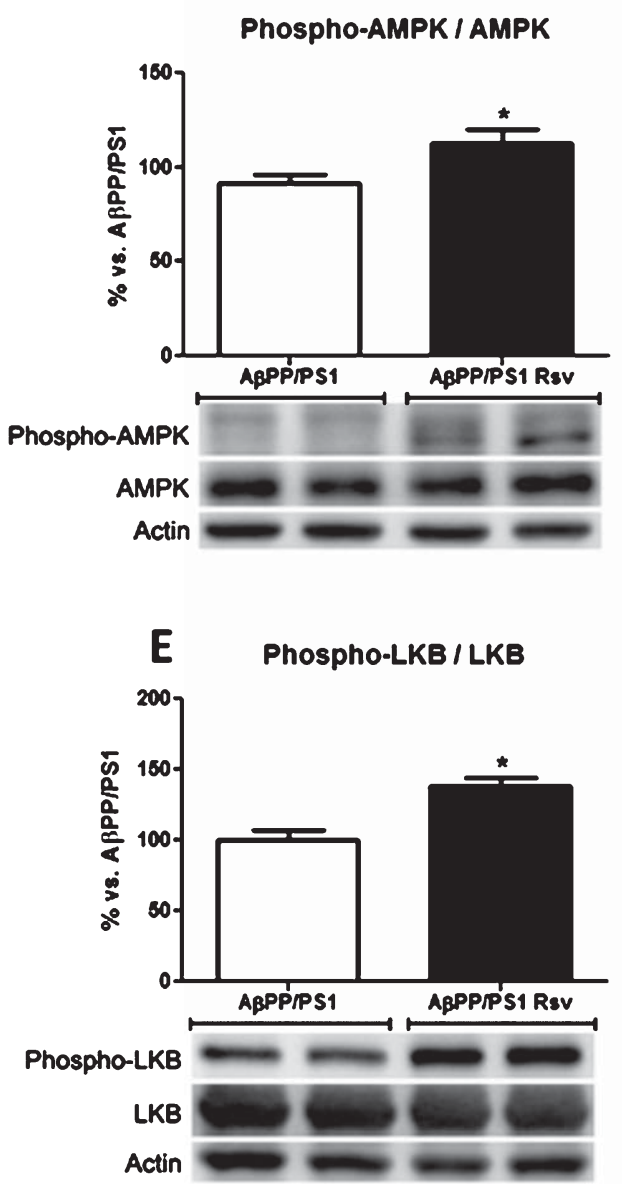

B

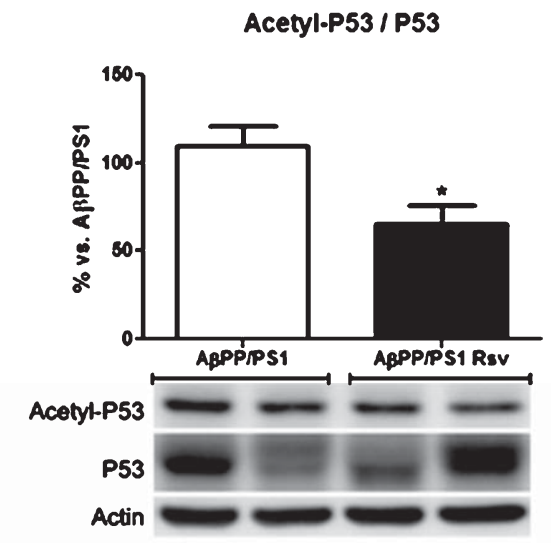

D

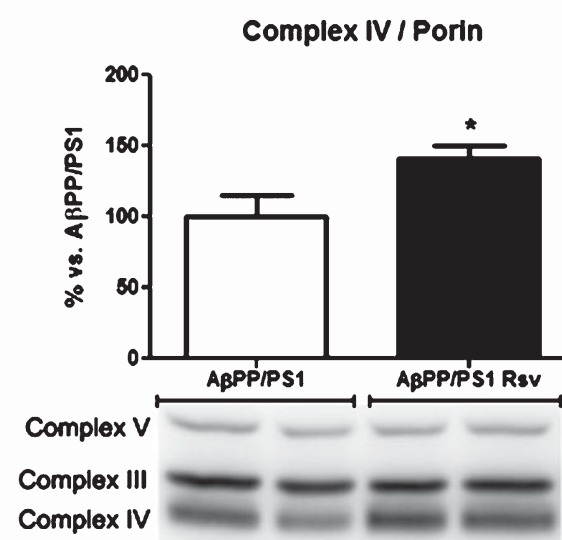

Complex II

Complex I

Porin

Actin

Fig. 4. Western blot analysis for Sirtuin 1 (SIRT1) (A), acetylated-p53 (B), phospho-5-Adenosine monophosphate (AMP)-activated protein kinase (p-AMPK) (C), mitochondrial complex IV (MCIV) (D), and serine-threonine kinase liver kinase B (LKB) (E). Bars represent mean \pm Standard error of the mean (SEM); Student $t$-test: ${ }^{*} p<0.05$ versus control. 
A II1b Expression (mRNA)

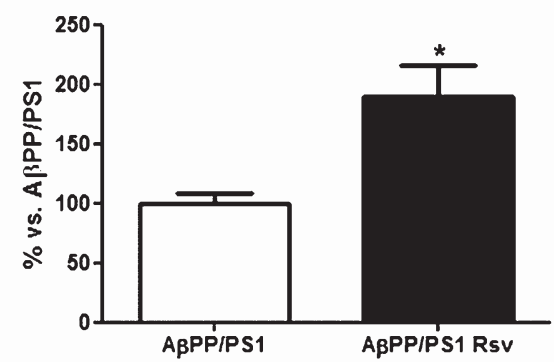

C

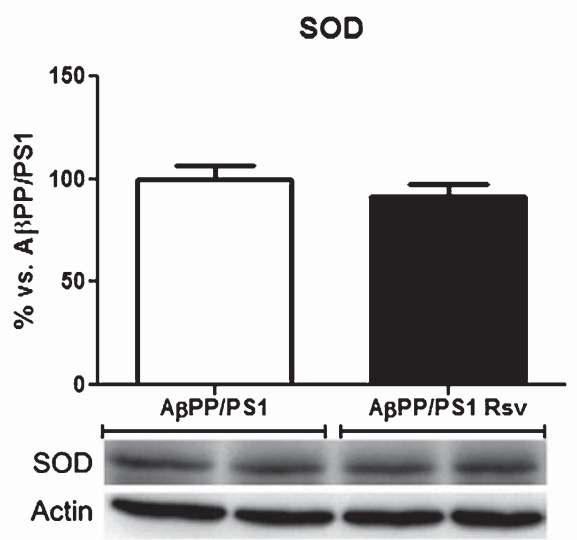

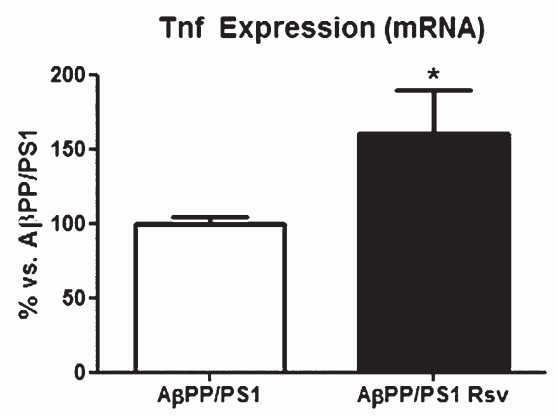

D

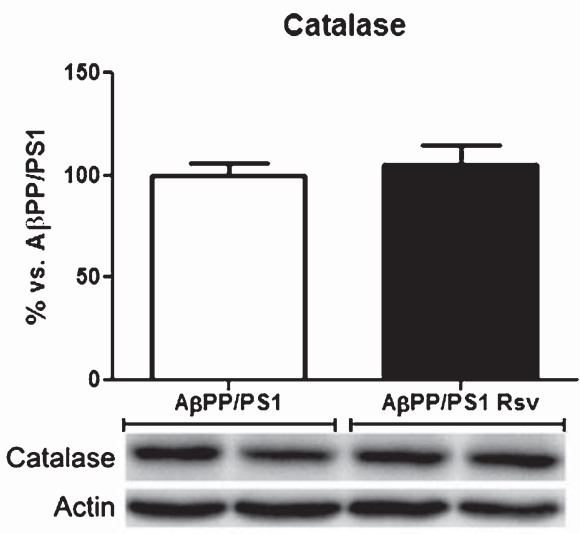

Fig. 5. Increases in gene expression for Interleukin-1 $\beta$ (IL1 $\beta$ ) (A) and tumor necrosis factor (TNF) (B). Western blot analysis for protein levels of superoxide dismutase (SOD) and catalase (CAT) (C, D). Bars represent mean \pm Standard error of the mean (SEM); Student $t$-test: ${ }^{*} p<0.05$ versus control.

did not alter levels of high-molecular-weight A $\beta P P$ (holo A $\beta$ PP) (Fig. 3D), $\beta$ CTF (C99) (Fig. 3E), or $\alpha$ CTF (C83) (Fig. 3F).

\section{Resveratrol treatment increases the} phospho-AMPK (p-AMPK)/LKB pathway in ARPP/PS1 although SIRT1 levels are decreased

Because previous studies demonstrated that resveratrol activates the nicotinamide adenine dinucleotide (NAD)-dependent protein deacetylase SIRT1, we tested whether the SIRT1 protein level was upregulated in A $\beta P P / P S 1$ mice fed with resveratrol. As demonstrated in Fig. 4, resveratrol treatment did not increase SIRT1, but a significant diminution in acetyl-p53, one of the main substrates of SIRT1, was observed (Fig. 4A, B). Moreover, p-AMPK and also p-LKB were significantly increased in mice fed resveratrol (Fig. 4C-E), indicating modulation by this polyphenol on phosphorylation, thus on the activity of these two kinases. In reference to mitochondria and according to stimulation of AMPK, an increase in MCIV expression, but not in other complexes, was determined after resveratrol treatment (Fig. 4D).

Effect of resveratrol treatment on cytokine gene expression and catalase/superoxide dismutase (CAT/SOD) protein levels in hippocampus

To test the effect of resveratrol on inflammation and oxidative stress, different markers for these were determined. Resveratrol feeding increased Ill $\mathrm{b}$ and Tnf mRNA expression (Fig. 5A, B). In contrast, no changes were determined in I16, Nfe212, Hmox, and Jun expression (data not shown). Protein levels of CAT and SOD were also unchanged (Fig. 5C, D).

\section{DISCUSSION}

A $\beta P P / P S 1$ mice express a chimeric mouse/human amyloid- $\beta$ protein precursor (Mo/Hu A $\beta$ PP695swe) and a mutant human presenilin 1 (PS1-dE9) that lead to 
altered $\mathrm{A} \beta \mathrm{PP}$ and $\mathrm{PS} 1$ proteins. Both cause $\mathrm{A} \beta$ plaques from 3 months onward, and they increase in number and distribution with disease progression in parallel with increased levels of brain-soluble $A \beta_{1-42}$ and $\mathrm{A} \beta_{1-40}$, but also with a reduced $A \beta_{1-42 / 1-40}$ ratio with age. Amyloid deposition in plaques is accompanied by altered mitochondria and increased oxidative damage, post-translational modifications, and the accumulation of altered proteins at the dystrophic neurites surrounding plaques [24]. This model is taken as a familial AD murine model. Resveratrol has been described as a natural compound with pleiotropic neuroprotective activities, linked with antioxidant properties and the modification of activity of different enzymatic pathways, such as the SIRT1 pathway, AMPK activation, or cellular protein degradation machinery [25].

A $\beta P$ PS 1 mice fed with resveratrol showed a significant decrease in the number and intensity of amyloid plaques, when were measured by specific $\mathrm{S}$ thioflavine staining. Reduction in plaque pathology did not appear to be due to altered A $\beta P P$ processing toward the non-amyloidogenic pathway, because highmolecular-weight A $\beta$ PP (holo A $\beta P P), C 99$ (Carboxy terminal fragment beta, CTF $\beta$ ), and C83 (Carboxy terminal fragment alpha, $\mathrm{CTF} \alpha$ ) were unaltered; this is in agreement with a study conducted by Karuppagounder and coworkers [26] on Tg19959 fed with resveratrol. However, the significant decrease in BACE levels would ultimately have altered the amyloidogenic pathway, leading to a reduction in amyloid burden found in A $\beta P P / P S 1$ fed for a lengthy period with resveratrol. Evidence is compelling that a decrease in proteasome activity occurs in AD brains [27, 28] and in AD murine models, including A $\beta P P / P S 1$, a familial AD model $[24,29]$, and in vitro, resveratrol promotes $A \beta$ clearance by increasing intracellular proteasomal activity without affecting $A \beta$ - producing enzyme activities $(\beta$ secretase and $\gamma$-secretase) [30]. This mechanism on the proteasomal system exerted by resveratrol could be synergistic to BACE reduction, explaining the decrease in plaques observed with resveratrol treatment, albeit than a decrease in ADAM10 was also determined.

Improvement of short-term memory in these mice was evaluated by the novel object recognition test (NORT), a test widely used to evaluate learning and memory $[24,31]$. The brain areas involved in this test are mainly hippocampus and perirhinal cortex. Moreover, NORT requires no external motivation, reward or punishment, thus avoiding the anxyogenic role in cognition impairment, although A $\beta P P / P S 1$ mice do not present anxiety behavior [32, 33]. Synaptophysin, an integral membrane glycoprotein present in presynaptic vesicles, was used to quantify synapse number. In our case, A $\beta P P / P S 1$ mice resveratrol fed increased synaptophysin levels, correlating with shortterm memory improvement and reduction in amyloid plaques.

Several reports suggest that resveratrol possesses antioxidant properties [34]; thus, a tantalizing alternative speculation is that resveratrol-induced reduction in plaques may be occurring through its effect on the cellular antioxidant machinery. We measured protein levels of CAT and SOD, but there were no changes observed. In relation to the expression of several genes related with oxidative stress and inflammation, there were no changes found in the $N f e 2 l 2$ and Hmoxl genes, which are implicated in neuroprotection mechanisms $[35,36]$, whereas cytokines linked with inflammation processes, such as IL1 $\beta$ and TNF $\alpha$, were increased. In $\mathrm{AD}$, abnormal accumulation of $\mathrm{A} \beta$ is thought to be intimately linked with the immune system [37]. The majority of efforts have been carried out in order to understand the role of activated astroglia and microglia, which secrete cytokines that act in favor of phagocytosis and A $\beta P P$ and $A \beta$ peptide clearance, giving rise to the hypothesis that slight increases in cytokines will be protective by removing $A \beta$ and reducing the plaque burden in brain. Our results indicate that at this time of treatment, there is no effective action of resveratrol on the inflammation and oxidative machinery of A $\beta P P / P S 1$ mice; the antioxidant effect could have occurred at an earlier treatment time, as described for another AD murine model by Karuppagounder and coworkers [26]. However, long-term resveratrol treatment allows fine control of antioxidant machinery by increasing IL $1 \beta$ and $\mathrm{TNF} \alpha$, leading to a hormetic process that ultimately yielded a reduction in plaque number and the beneficial effect on memory, as we observed clearly in this familal AD mouse model at a late age. This fact cannot rule out the possibility that resveratrol exerts an earlier antioxidant effect at younger ages, acting as a preventive more than as a curative agent.

In addition, resveratrol has been postulated to have its beneficial effects on the lifespan, neurodegeneration, and memory improvement by activation of SIRT1 $[38,39]$. Because SIRT1 activation is one of the main targets defined for the pharmacological effects of resveratrol, the levels of this deacetylase were determined. Here we observed a diminution in SIRT1 protein levels, but a decrease in acetyl-p53 was determined concomitantly. These results are in agreement 
with the literature in other transgenic models and in in vitro experiments where a reduction in plaque pathology without increase in sirtuin levels [26] but decrease in PGC1 $\alpha$ acetylation was found [39]. The decline in SIRT1, together with p53 deacetylation status, could reflect decreased synthesis of this deacetylase, but increased activity mediated by resveratrol [40-42] or by crossregulation with the AMPK/LKB pathway [34, 43, 44].

Cantó and Auwerx [45] demonstrated that activation of AMPK stimulated the functional activity of SIRT1 by increasing the intracellular concentration of NAD+. Interestingly, SIRT1 was able to deacetylate LKB1 kinase, which subsequently increased its activity [46]. Because LKB1 is an upstream activator of AMPK, this signaling pathway stimulates the activation of AMPK. This positive feedback loop between SIRT1 and AMPK can also potentiate the function of the other AMPK-activated signaling pathways [47]. In the current study, the surprising resveratrol-induced decline in SIRT1 levels is consistent with AMPK/LKB activation.

The AMPK and SIRT1 signaling pathways are highly conserved energy sensors of increased levels of AMP and NAD+, respectively, and AMPK signaling is involved in the regulation of energy metabolic homeostasis [48]. In line with this, resveratrol also normalized mitochondrial function and enhanced mitochondrial biogenesis in the spinal cord of SOD1 ${ }^{\mathrm{G} 93 \mathrm{~A}}$ amyotrophic lateral sclerosis mice [49]. The majority of the pathways mentioned previously are involved in mitochondrial function, and in this particular organelle, the A $\beta P P / P S 1$ life-long treatment with resveratrol induced an increase in complex IV. MCIV is considered a marker of mitochondrial functionality $[4,5]$, which in turn is known to be decreased in AD. In our study, resveratrol increased MCIV, because this action can be taken in account for delineating the neuroprotective role of resveratrol in A $\beta P P / P S 1$ mice.

In conclusion, this study supports some unexplored pathways responsible for the neuroprotective effects of resveratrol in A $\beta P P / P S 1$ mice, namely, the equilibrium among SIRT1 and AMPK signaling, mitochondrial status, and inflammatory changes. In addition to other reports $[26,50]$, our findings indicate that the onset of this neurodegenerative disease may be delayed or mitigated employing dietary resveratrol, which is able to protect against $\mathrm{A} \beta$ plaque formation and cognitive loss. Further studies need to be conducted to elucidate the precise regulatory mechanisms that can be modulated by resveratrol in the A $\beta P P / P S 1$ mouse model.

\section{ACKNOWLEDGMENTS}

We thank Maggie Brunner, M.A., for revising the language and style of the manuscript. This study was supported by grants SAF-2011-23631 and SAF2012-39852 from the "Ministerio de Educación y Ciencia" and 2014/SGR/525 from the "Generalitat de Catalunya".

Authors' disclosures available online (http://www.jalz.com/disclosures/view.php?id=2320).

\section{REFERENCES}

[1] Donmez G, Wang D, Cohen DE, Guarente L (2010) SIRT1 suppresses beta-amyloid production by activating the alphasecretase gene ADAM10. Cell 142, 320-332.

[2] Lin MT, Beal MF (2006) Mitochondrial dysfunction and oxidative stress in neurodegenerative diseases. Nature 443 , 787-795.

[3] Maurer I, Zierz S, Möller HJ (2000) A selective defect of cytochrome c oxidase is present in brain of Alzheimer disease patients. Neurobiol Aging 21, 455-462.

[4] Nunomura A, Castellani RJ, Zhu X, Moreira PI, Perry G, Smith MA (2006) Involvement of oxidative stress in Alzheimer disease. J Neuropathol Exp Neurol 65, 631-641.

[5] Sultana R, Mecocci P, Mangialasche F, Cecchetti R, Baglioni M, Butterfield DA (2011) Increased protein and lipid oxidative damage in mitochondria isolated from lymphocytes from patients with Alzheimer's disease: Insights into the role of oxidative stress in Alzheimer's disease and initial investigations into a potential biomarker for this. J Alzheimers Dis $\mathbf{2 4}$, 77-84.

[6] Reddy PH, Beal MF (2008) Amyloid beta, mitochondrial dysfunction and synaptic damage: Implications for cognitive decline in aging and Alzheimer's disease. Trends Mol Med 14, 45-53.

[7] Beaudoin M-S, Snook LA, Arkell AM, Simpson JA, Holloway GP, Wright DC (2013) Resveratrol supplementation improves white adipose tissue function in a depot-specific manner in Zucker diabetic fatty rats. Am J Physiol Regul Integr Comp Physiol 305, 542-551.

[8] Estrela JM, Ortega A, Mena S, Rodriguez ML, Asensi M (2013) Pterostilbene: Biomedical applications. Crit Rev Clin Lab Sci 50, 65-78.

[9] Jayasena T, Poljak A, Smythe G, Braidy N, Münch G, Sachdev $\mathrm{P}$ (2013) The role of polyphenols in the modulation of sirtuins and other pathways involved in Alzheimer's disease. Ageing Res Rev 12, 867-883.

[10] Um J-H, Park S-J, Kang H, Yang S, Foretz M, McBurney MW, Kim MK, Viollet B, Chung JH (2010) AMP-activated protein kinase-deficient mice are resistant to the metabolic effects of resveratrol. Diabetes 59, 554-563.

[11] De Lange P, Farina P, Moreno M, Ragni M, Lombardi A, Silvestri E, Burrone L, Lanni A, Goglia F (2006) Sequential changes in the signal transduction responses of skeletal muscle following food deprivation. FASEB J 20, 2579-2581.

[12] Garcia-Alloza M, Robbins EM, Zhang-Nunes SX, Purcell SM, Betensky RA, Raju S, Prada C, Greenberg SM, Bacskai BJ, Frosch MP (2006) Characterization of amyloid deposition in the APPswe/PS1dE9 mouse model of Alzheimer disease. Neurobiol Dis 24, 516-524. 
[13] Huang HJ, Liang KC, Ke HC, Chang YY, Hsieh-Li HM (2011) Long-term social isolation exacerbates the impairment of spatial working memory in APP/PS1 transgenic mice. Brain Res 1371, 150-160.

[14] Minkeviciene R, Ihalainen J, Malm T, Matilainen O, KeksaGoldsteine V, Goldsteins G, Iivonen H, Leguit N, Glennon J, Koistinaho J, Banerjee P, Tanila HJ (2008) Age-related decrease in stimulated glutamate release and vesicular glutamate transporters in APP/PS1 transgenic and wild-type mice. Neurochem 105, 584-594.

[15] Savonenko A, Xu GM, Melnikova T, Morton JL, Gonzales V, Wong MP, Price DL, Tang F, Markowska AL, Borchelt DR (2005) Episodic-like memory deficits in the APPswe/PS1dE9 mouse model of Alzheimer's disease: Relationships to beta-amyloid deposition and neurotransmitter abnormalities. Neurobiol Dis 18, 602-617.

[16] Webster SJ, Bachstetter AD, Van Eldik LJ (2013) Comprehensive behavioral characterization of an APP/PS-1 double knock-in mouse model of Alzheimer's disease. Alzheimers Res Ther 5, 28.

[17] Torres M, Jimenez S, Sanchez-Varo R, Navarro V, TrujilloEstrada L, Sanchez-Mejias E, Carmona I, Davila JC, Vizuete M, Gutierrez A, Vitorica J (2012) Defective lysosomal proteolysis and axonal transport are early pathogenic events that worsen with age leading to increased APP metabolism and synaptic Abeta in transgenic APP/PS1 hippocampus. Mol Neurodegener 7, 59.

[18] Reinhardt S, Schuck F, Grösgen S, Riemenschneider M, Hartmann T, Postina R, Grimm M, Endres K (2014) Unfolded protein response signaling by transcription factor XBP-1 regulates ADAM10 and is affected in Alzheimer's disease. FASEB J 28, 978-997.

[19] Pérez-González R, Alvira-Botero MX, Robayo O, Antequera D, Garzón M, Martín-Moreno AM, Brera B, de Ceballos ML, Carro E (2014) Leptin gene therapy attenuates neuronal damages evoked by amyloid- $\beta$ and rescues memory deficits in APP/PS1mice. Gene Ther 21, 298-308.

[20] Yan JJ, Jung JS, Kim TK, Hasan A, Hong CW, Nam JS, Song DK (2013) Protective effects of ferulic acid in amyloid precursor protein plus presenilin-1 transgenic mouse model of Alzheimer disease. Biol Pharm Bull 36, 140-143.

[21] Wang TH, Jiang Y, Xiao LP (2013) Expression of amyloid beta-protein in bone tissue of APP/PS1 transgenic mouse. Zhonghua Yi Xue Za Zhi 93, 65-68.

[22] Porquet D, Casadesús G, Bayod S, Vicente A, Canudas AM, Vilaplana J, Pelegrí C, Sanfeliu C, Camins A, Pallás M, del Valle J (2013) Dietary resveratrol prevents Alzheimer's markers and increases life span in SAMP8. Age (Dordr) 35, 1851-1865.

[23] Puighermanal E, Marsicano G, Busquets-Garcia A, Lutz B, Maldonado R, Ozaita A (2009) Cannabinoid modulation of hippocampal long-term memory is mediated by mTOR signaling. Nat Neurosci 12, 1152-1158.

[24] Aso E, Lomoio S, López-González I, Joda L, Carmona M, Fernández-Yagüe N, Moreno J, Juvés S, Pujol A, Pamplona R, Portero-Otin M, Martín V, Díaz M, Ferrer I (2012) Amyloid generation and dysfunctional immunoproteasome activation with disease progression in animal model of familial Alzheimer's disease. Brain Pathol 22, 636-653.

[25] Pallás M, Porquet D, Vicente A, Sanfeliu C (2013) Resveratrol: New avenues for a natural compound in neuroprotection. Curr Pharm Des 19, 6726-6731.

[26] Karuppagounder SS, Pinto JT, Xu H, Chen H-L, Beal MF, Gibson GE (2009) Dietary supplementation with resver- atrol reduces plaque pathology in a transgenic model of Alzheimer's disease. Neurochem Int 54, 111-118.

[27] Keller JN, Hanni KB, Markesbery WR (2000) Impaired proteasome function in Alzheimer's disease. J Neurochem $\mathbf{7 5}$, 436-439.

[28] López Salon M, Morelli L, Castaño EM, Soto EF, Pasquini JM (2000) Defective ubiquitination of cerebral proteins in Alzheimer's disease. J Neurosci Res 15, 302-310.

[29] Van Tijn P, Dennissen FJA, Gentier RJG, Hobo B, Hermes D, Steinbusch HWM, Van Leeuwen FW, Fischer DF (2012) Mutant ubiquitin decreases amyloid $\beta$ plaque formation in a transgenic mouse model of Alzheimer's disease. Neurochem Int 61, 739-748.

[30] Marambaud P, Zhao H, Davies P (2005) Resveratrol promotes clearance of Alzheimer's disease amyloid-beta peptides. $J$ Biol Chem 280, 37377-37382.

[31] Antunes M, Biala G (2012) The novel object recognition memory: Neurobiology, test procedure, and its modifications. Cogn Process 13, 93-110.

[32] Webster SJ, Bachstetter AD, Van Eldik LJ (2013) Comprehensive behavioral characterization of an APP/PS-1 double knock-in mouse model of Alzheimer's disease. Alzheimers Res Ther 5, 28.

[33] Lok K, Zhao H, Zhang C, He N, Shen H, Wang Z, Zhao W, Yin $M$ (2013) Effects of accelerated senescence on learning and memory, locomotion and anxiety-like behavior in APP/PS1 mouse model of Alzheimer's disease. J Neurol Sci 335, 145154.

[34] Dasgupta B, Milbrandt J (2007) Resveratrol stimulates AMP kinase activity in neurons. Proc Natl Acad Sci U S A 104, 7217-7222.

[35] Quincozes-Santos A, Bobermin LD, Tramontina AC, Wartchow KM, Tagliari B, Souza DO, Wyse AT, Gonçalves CA (2014) Oxidative stress mediated by NMDA, AMPA/KA channels in acute hippocampal slices: Neuroprotective effect of resveratrol. Toxicol In Vitro 28, 544-551.

[36] Son Y, Byun SJ, Pae HO (2013) Involvement of heme oxygenase-1 expression in neuroprotection by piceatannol, a natural analog and a metabolite of resveratrol, against glutamate-mediated oxidative injury in HT22 neuronal cells. Amino Acids 45, 393-401.

[37] Serpente M, Bonsi R, Scarpini E, Galimberti D (2014) Innate immune system and inflammation in Alzheimer's disease: From pathogenesis to treatment. Neuroimmunomodulation 21, 79-87.

[38] Kim D, Nguyen MD, Dobbin MM, Fischer A, Sananbenesi F, Rodgers JT, Delalle I, Baur JA, Sui G, Armour SM, Puigserver P, Sinclair DA, Tsai LH (2007) SIRT1 deacetylase protects against neurodegeneration in models for Alzheimer's disease and amyotrophic lateral sclerosis. EMBO J 26, 3169-3179.

[39] Lagouge M, Argmann C, Gerhart-Hines Z, Meziane H, Lerin C, Daussin F, Messadeq N, Milne J, Lambert P, Elliott P, Geny B, Laakso M, Puigserver P, Auwerx J (2006) Resveratrol improves mitochondrial function and protects against metabolic disease by activating SIRT1 and PGC-1alpha. Cell 127, 1109-1122.

[40] Della-Morte D, Dave KR, DeFazio RA, Bao YC, Raval AP, Perez-Pinzon MA (2009) Resveratrol pretreatment protects rat brain from cerebral ischemic damage via a sirtuin 1uncoupling protein 2 pathway. Neuroscience 159, 993-1002.

[41] Du L-L, Xie J-Z, Cheng X-S, Li X-H, Kong F-L, Jiang X, Ma ZW, Wang JZ, Chen C, Zhou XW (2014) Activation of sirtuin 1 attenuates cerebral ventricular streptozotocin-induced tau hyperphosphorylation and cognitive injuries in rat hippocampi. Age (Dordr) 36, 613-623. 
[42] Kaeberlein M, McDonagh T, Heltweg B, Hixon J, Westman EA, Caldwell SD, Napper A, Curtis R, DiStefano PS, Fields S, Bedalov A, Kennedy BK (2005) Substrate-specific activation of sirtuins by resveratrol. J Biol Chem 280, 17038-17045.

[43] Hwang J-T, Kwak DW, Lin SK, Kim HM, Kim YM, Park OJ (2007) Resveratrol induces apoptosis in chemoresistant cancer cells via modulation of AMPK signaling pathway. Ann $N$ Y Acad Sci 1095, 441-448.

[44] Zang M, Xu S, Maitland-Toolan KA, Zuccollo A, Hou X, Jiang B, Wierzbicki M, Verbeuren TJ, Cohen RA (2006) Polyphenols stimulate AMP-activated protein kinase, lower lipids, and inhibit accelerated atherosclerosis in diabetic LDL receptor-deficient mice. Diabetes 55, 2180-2191.

[45] Cantó C, Auwerx J (2009) PGC-1alpha, SIRT1 and AMPK, an energy sensing network that controls energy expenditure. Curr Opin Lipidol 20, 98-105.

[46] Lan F, Cacicedo JM, Ruderman N, Ido Y (2008) SIRT1 modulation of the acetylation status, cytosolic localization, and activity of LKB1. Possible role in AMP-activated protein kinase activation. J Biol Chem 283, 27628-27635.

[47] Duan W (2013) Sirtuins: From metabolic regulation to brain aging. Front Aging Neurosci $\mathbf{5}, 36$.

[48] Hardie DG, Ross FA, Hawley SA (2012) AMPK: A nutrient and energy sensor that maintains energy homeostasis. Nat Rev Mol Cell Biol 13, 251-262.

[49] Mancuso R, Del Valle J, Modol L, Martinez A, GranadoSerrano AB, Ramirez-Núñez O, Pallás M, Portero-Otin M, Osta R, Navarro X (2014) Resveratrol improves motoneuron function and extends survival in SOD1(G93A) ALS mice. Neurotherapeutics 11, 419-432.

[50] Wang J, Ho L, Zhao Z, Seror I, Humala N, Dickstein DL, Thiyagarajan M, Percival SS, Talcott ST, Pasinetti GM (2006) Moderate consumption of Cabernet Sauvignon attenuates Abeta neuropathology in a mouse model of Alzheimer's disease. FASEB J 20, 2313-2320. 